McGill Law Journal

Revue de droit de McGill

\title{
Charles Gonthier : l'homme derrière le juriste
}

Jacques-Yvan Morin

Volume 55, numéro 2, july 2010

URI : https://id.erudit.org/iderudit/045089ar

DOI : https://doi.org/10.7202/045089ar

Aller au sommaire du numéro

Éditeur(s)

McGill Law Journal / Revue de droit de McGill

ISSN

0024-9041 (imprimé)

1920-6356 (numérique)

Découvrir la revue

Citer cet article

Morin, J.-Y. (2010). Charles Gonthier : l'homme derrière le juriste. McGill Law Journal / Revue de droit de McGill, 55(2), 333-335.

https://doi.org/10.7202/045089ar d'utilisation que vous pouvez consulter en ligne.

https://apropos.erudit.org/fr/usagers/politique-dutilisation/ 
McGill Law Journal Revue de droit de McGill

\section{CHARLES GONTHIER : L'HOMME DERRIÈRE LE JURISTE}

\section{Jacques-Yvan Morin ${ }^{*}$}

Ce n'est pas sans émotion que j'ai accepté d'évoquer la personnalité de Charles Gonthier. Dans notre jeunesse, nous faisions partie d'un groupe d'amis qui existe toujours, en dépit des pertes que nous avons subies. Aussi voudrais-je m'attacher avant tout à parler de l'homme plutôt que d'analyser les nombreux arrêts qu'il fut appelé à rendre ou auxquels il participa dans ses fonctions judiciaires. Certes, on ne saurait prédire exactement le destin des êtres dès leur jeune âge, mais il est permis de constater a posteriori que les qualités dont ils ont fait preuve et le tempérament qui s'est révélé étaient déjà présents à l'adolescence et ont servi de fondement aux idées et convictions qui ont guidé leur vie et, dans le cas de Charles, inspiré sa contribution au progrès du droit.

Les modèles n'ont pas manqué, à commencer par la troupe scoute dont nous faisions partie. Guidés par les mêmes chefs, nous avons appris le dévouement, la discipline et le sens des responsabilités. Toutes ces valeurs et aptitudes collaient à la personnalité de Charles et me paraissent avoir inspiré ses réflexions sur la place de la fraternité, de la liberté et de l'égalité dans les fondements de la règle juridique. À cela s'ajoute la formation classique à la française reçue au Collège Stanislas de Montréal, "Stan" pour ses anciens. Bien qu'appartenant à des promotions différentes, nous y avons eu les mêmes maîtres, dont plusieurs de qualité exceptionnelle. Tout en s'appuyant sur les principes chrétiens qui ont servi de base à la fondation du Collège, l'enseignement de la philosophie n'hésitait pas à montrer la diversité des systèmes de pensée et des croyances ainsi que le respect dû aux convictions d'autrui. Ces habitudes intellectuelles ne seront pas oubliées ; elles tombaient en bonne terre.

Cette formation classique a bien préparé Charles Gonthier aux études en droit à l'Université McGill. Nous y avons eu des maîtres comme Frank Scott, Louis Baudouin et Maxwell Cohen, qui ne manquaient pas

* Professeur émérite de la Faculté de droit de l'Université de Montréal, membre de l'Institut de droit international, ancien rédacteur en chef de la Revue de droit de McGill.

(C) Jacques-Yvan Morin 2010

Citation: (2010) 55 McGill L.J. $333 \sim$ Référence : (2010) 55 R.D. McGill 333 
d'assurer la diversité des points de vue. Tous ceux qui l'ont vu à l'œuvre à cette époque, se souviendront de l'application exemplaire de Charles dans ses études, qui faisait de lui un premier de classe naturel, tant au collège qu'à l'université. Sa solide et sérieuse formation académique constitue le pilier qui lui a permis de gravir tous les échelons de la profession juridique, comme avocat dans une étude dont nous avons tous deux été membres, puis dans la magistrature, au sein de laquelle son parcours exceptionnel devait le conduire à la Cour suprême du Canada.

Le côté humain de Charles présente aussi des traits moins connus. Notre groupe d'amis s'est transformé peu à peu en équipe de danse folklorique, dont faisaient partie des femmes et des hommes qui ont également laissé leur trace, tels le cinéaste Claude Jutras, la peintre Suzanne Joubert et l'écrivaine Hélène Pelletier-Baillargeon. Ces activités de nos jeunes années, en marge de nos études, alliaient les danses traditionnelles de plusieurs pays à celles du terroir, sans exclure les pas plus modernes (ceux de l'époque naturellement). Ces activités du vendredi soir ont exigé pendant plusieurs années discipline et esprit d'équipe et Charles s'y appliquait avec la même assiduité et cette soif d'apprendre qu'il sut conserver toute sa vie, dans les petites choses comme dans les grandes.

Le portrait du jeune homme serait incomplet sans mentionner les convictions religieuses qui étaient les siennes. Chrétien fervent, il était arrivé à l'âge d'homme avec une haute conception des exigences de la morale, pour lui-même au premier chef. Le respect de l'autre, dont il s'était pénétré, cependant, n'était pas compatible à ses yeux avec un système de normes éthiques unique ou exclusif que le droit aurait pour mission de consacrer. Dans une société de plus en plus diversifiée dans ses origines et ses croyances, l'État doit certes protéger les valeurs fondamentales de la morale, mais il doit agir, en démocratie, en recherchant les dénominateurs communs. Cette attitude reflète fidèlement le sens de la mesure qui caractérisait Charles Gonthier et son souci du consensus. Cette dimension de l'homme éclaire sa philosophie du droit.

Le souci de l'autre l'amenait à mettre en évidence, à côté de la liberté et de l'égalité, un troisième pilier du droit, trop souvent occulté, même dans la Charte canadienne des droits et libertés ${ }^{1}$ et la Charte des droits et libertés de la personne du Québec ${ }^{2}$ : la fraternité. Ces trois principes fondamentaux vont de pair. Cette idée, qui plonge ses racines dans la formation qu'il a reçue autrefois, nourrie par l'expérience acquise en tant que juge, a beaucoup occupé ses pensées depuis quelques années. Les écrits et

1 Partie I de la Loi constitutionnelle de 1982, constituant l'annexe B de la Loi de 1982 sur le Canada (R.-U.), 1982, app. II, nº 2.

2 Charte des droits et libertés de la personne, L.R.Q. c. C-12. 
opinions qu'il y a consacrés font partie de l'héritage qu'il nous laisse : la recherche patiente des valeurs communes et de la solidarité dans une société complexe.

Charles avait le sens de la dignité individuelle et le souci de protéger les libertés fondamentales dans une société qui a adopté diverses chartes ou déclarations des droits depuis quelque trente-cinq ans, mais il estimait que ces libertés devaient s'inscrire dans une communauté envers laquelle chacun a des devoirs. À ses yeux, droits et responsabilités sont indissociables, ce qui nous ramène à la fraternité comme condition de la cohésion sociale, objet constant de ses préoccupations.

La retraite n'a guère modifié les activités intellectuelles de ce juriste accompli. Des opinions judiciaires, il est passé aux conférences, ce qui lui a permis de continuer sa quête des valeurs communes et de faire une synthèse de ses idées sur la liberté, la solidarité, la fraternité, la complémentarité des droits et devoirs, la moralité nécessaire à la cohésion sociale et la protection des droits collectifs des minorités et des autochtones. Depuis quelques années, il s'intéressait davantage à la dimension internationale des problèmes juridiques et participait à divers forums qui stimulaient sa réflexion : les principes et valeurs qu'il avait dégagés dans le cadre de la communauté étatique n'étaient-ils pas applicables dans la collectivité plus large que forment les États? Sa volonté de poursuivre son activité intellectuelle a également pris la forme de sa participation au sein du Conseil consultatif de la Revue de droit de McGill.

Ainsi, la vie féconde de Charles Gonthier, comme celle de tout homme sans doute, a sa source dans ses jeunes années. Les qualités dont il a fait preuve tout au long de sa carrière, soit la modestie, la recherche du dialogue et la courtoisie dans toute discussion, des qualités liées à la fois à son tempérament réservé et à la fermeté de ses convictions, étaient déjà les siennes à l'adolescence. J'ai eu le privilège de le connaître à cette époque et les années qui ont suivi n'ont fait que confirmer la haute estime que j'ai toujours eu pour lui, comme d'ailleurs tous ceux qui l'ont fréquenté.

Malgré des cheminements ultérieurs différents et des opinions pas toujours partagées, nous cultivions cette vieille amitié. Celles qui naissent dans la jeunesse sont sans doute les plus durables. 